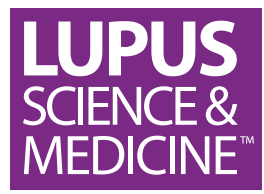

\title{
Incidence rates of systemic lupus erythematosus in the USA: estimates from a meta-analysis of the Centers for Disease Control and Prevention national lupus registries
}

Peter M Izmirly (D) , ${ }^{1}$ Elizabeth D Ferucci (D) , ${ }^{2}$ Emily C Somers (D) , ${ }^{3}$ Lu Wang, ${ }^{4}$ S Sam Lim (1) , ${ }^{5}$ Cristina Drenkard (1) , ${ }^{5}$ Maria Dall'Era, ${ }^{6}$ W Joseph McCune, ${ }^{7}$ Caroline Gordon, ${ }^{8}$ Charles Helmick, ${ }^{9}$ Hilary Parton ${ }^{10}$
To cite: Izmirly PM, Ferucci ED, Somers EC, et al. Incidence rates of systemic lupus erythematosus in the USA: estimates from a metaanalysis of the Centers for Disease Control and Prevention national lupus registries. Lupus Science \& Medicine 2021;8:e000614. doi:10.1136/ lupus-2021-000614

Received 11 November 2021 Accepted 25 November 2021
Check for updates

(c) Author(s) (or their employer(s)) 2021. Re-use permitted under CC BY-NC. No commercial re-use. See rights and permissions. Published by BMJ.

For numbered affiliations see end of article.

Correspondence to Dr Peter M Izmirly; Peter. Izmirly@nyumc.org

\section{ABSTRACT}

Objective To estimate the annual incidence rate of SLE in the USA

Methods A meta-analysis used sex/race/ethnicityspecific data spanning 2002-2009 from the Centers for Disease Control and Prevention network of four population-based state registries to estimate the incidence rates. SLE was defined as fulfilling the 1997 revised American College of Rheumatology classification criteria. Given heterogeneity across sites, a random effects model was employed. Applying sex/race/ethnicity-stratified rates, including data from the Indian Health Service registry, to the 2018 US Census population generated estimates of newly diagnosed SLE cases.

Results The pooled incidence rate per 100000 personyears was 5.1 (95\% Cl 4.6 to 5.6), higher in females than in males (8.7 vs 1.2), and highest among black females (15.9), followed by Asian/Pacific Islander (7.6), Hispanic (6.8) and white (5.7) females. Male incidence was highest in black males (2.4), followed by Hispanic (0.9), white (0.8) and Asian/Pacific Islander (0.4) males. The American Indian/Alaska Native population had the second highest race-specific SLE estimates for females (10.4 per 100 000) and highest for males (3.8 per 100 000). In 2018, an estimated 14263 persons ( $95 \% \mathrm{Cl} 11563$ to 17 735) were newly diagnosed with SLE in the USA.

Conclusions A network of population-based SLE registries provided estimates of SLE incidence rates and numbers diagnosed in the USA.

\section{INTRODUCTION}

The heterogeneity of clinical manifestations of SLE, lack of a singular diagnostic test, racial/ethnic disparities in SLE susceptibility and mortality, differing case definitions, heterogeneous sources for case ascertainment, possible inaccuracy of self-report, unreliability in coding in health system databases, and variable access to healthcare for high-risk populations have made SLE difficult

\section{Key messages}

What is already known about this subject?

- Current epidemiological incidence estimates for SLE vary widely in the USA, especially among certain demographic groups.

What does this study add?

- Using the Centers for Disease Control and Prevention-supported network of five populationbased SLE registries that employed standardised active surveillance methods to determine SLE incidence in populations reflecting the broad distribution of racial/ethnic demographics in the USA, a metaanalysis to estimate the general and sex-specific and race/ethnicity-specific incidence rates of SLE was performed.

- Overall SLE incidence in the USA was estimated to be 5.1 per 100000 person-years $(95 \% \mathrm{Cl} 4.6$ to 5.6$)$ during calendar years 2002-2009.

- Incidence rates were approximately seven times higher in females compared with males and highest among black and American Indian/Alaska Native females.

- Extrapolating sex-specific and race-specific estimates to 2018 US Census data, we estimated that 14263 (95\% Cl 11563 to 17 735) persons (12560 and 1703 females and males, respectively) in the USA were newly diagnosed with SLE fulfilling the American College of Rheumatology classification criteria.

How might this impact on clinical practice or future developments?

- The data provide new information to inform future research and policy efforts addressing the burden of SLE.

for epidemiologists to study in the USA and resulted in widely varying epidemiological estimates. $^{1-3}$ 


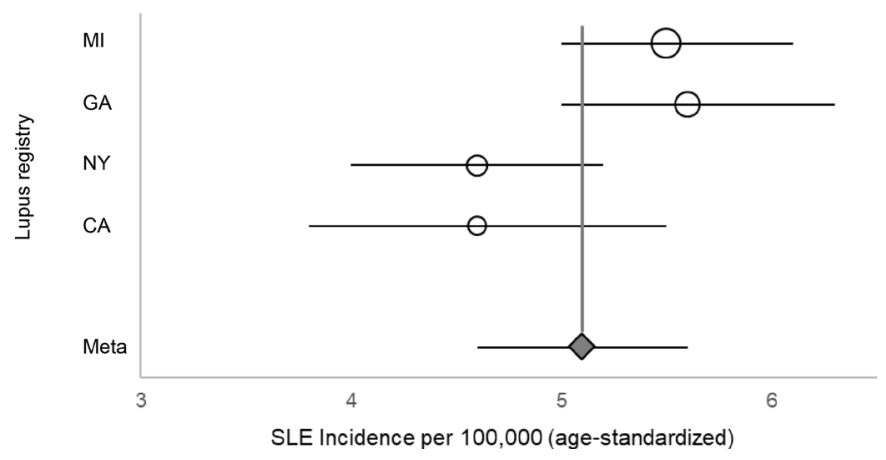

Figure 1 Meta-analysis results of the SLE incidence based on four CDC population-based registries, overall and by registry site. Overall incidence estimates for the SLE registry sites in Michigan (MI), Georgia (GA), New York (NY) and California (CA) are represented by circles, with the size of the circle corresponding to the weight of the contribution to the meta-analysis, and the diamond representing the results from the meta-analysis and the lines corresponding to $95 \%$ Cl. SLE cases were defined according to the 1997 revised American College of Rheumatology criteria. CDC, Centers for Disease Control and Prevention.

The Centers for Disease Control and Prevention (CDC) supported a network of five population-based SLE registries using standardised active surveillance methods to determine SLE prevalence and incidence in populations reflecting the broad distribution of $\mathrm{racial} / \mathrm{ethnic}$ demographics in the USA. These regional registries provided overall prevalence and incidence of SLE, as well as estimates that focused on the major US demographic groups. ${ }^{3-7}$ A meta-analysis using these registries provided overall estimates of SLE prevalence and the number of individuals diagnosed with SLE in the USA in $2018 .{ }^{8}$ Further leveraging these registries, a meta-analysis to estimate the general and sex-specific and race/ethnicityspecific incidence rates of SLE was performed and used to provide an estimate of the number of newly diagnosed SLE cases in the USA in 2018.

\section{METHODS}

\section{Data sources and study selection}

The methodology for this paper mirrored that used in our meta-analysis of the prevalence of SLE. ${ }^{8}$ The CDCsupported lupus registries included two sites with large black and white populations (Georgia Lupus Registry (GLR), Fulton and DeKalb counties ${ }^{3}$; Michigan Lupus Epidemiology and Surveillance Program (MILES), Washtenaw and Wayne counties ${ }^{4}$ ); two sites with large Hispanic and Asian/Pacific Islander populations (California Lupus Surveillance Program (CLSP), San Francisco county ${ }^{5}$; Manhattan Lupus Surveillance Program (MLSP), New York County) ${ }^{6}$ ); and one system to capture the American Indian/ Alaska Native (AI/AN) population (Indian Health Service (IHS), including facilities in Alaska, Phoenix and Oklahoma City areas ${ }^{7}$ ). These registries evaluated incident and prevalent cases occurring in 2002-2009 by performing active surveillance at various times between 2003 and 2015 as previously described. ${ }^{3-7}$ The case definitions for SLE incidence varied slightly by the time period evaluated in each registry, with all taking place between 2002 and 2009..$^{3-7}$ The primary case definition for SLE was the 1997 revised American College of Rheumatology (ACR) classification criteria for SLE. ${ }^{910}$ The registries used harmonised methods including screening for potential SLE cases using the same core set of International Classification of Diseases, Ninth Revision (ICD-9) codes and a variety of case-finding sources and a consistent approach to capture the relevant clinical and demographic information and core definitions from a standardised data dictionary. Medical abstractors, who underwent routine training and quality assurance monitoring, collected the data. Denominators were based on intercensal population estimates for the respective source populations. Sex-specific and race/ethnicityspecific incidence estimates were calculated per 100000 person-years and age-adjusted to the 2000 US standard population. ${ }^{11}$ Data were extracted by two authors independently (PI and HP) from published manuscripts, who agreed on all data used.

\section{Data synthesis and analysis}

A meta-analysis derived pooled incidence estimates using data from four state-based, CDC-funded registries (GLR, MILES, CLSP and MLSP) for agestandardised incidence rates $^{11}$ and for rates stratified by sex and race/ethnicity categories, with the exception of the AI/AN population. ${ }^{3-6}$ Given that the IHS-based registry ${ }^{7}$ was different, focusing on one demographic population (AI/AN), it was handled separately. For the meta-analysis, heterogeneity across the four state-based sites was tested by Cochran's $Q$ and $\mathrm{I}^{2}$ statistics. ${ }^{12}{ }^{13}$ Due to significant heterogeneity, a random effects model, weighted by the population denominator for each site, was used to calculate the pooled incidence, ${ }^{14}$ which allowed for an underlying distribution of the effect sizes across different studies. Pooled sex-specific and race/ethnicity-specific estimates were calculated, except for the AI/AN estimate, which used the previously published female age-adjusted incidence estimates ${ }^{7}$ and newly calculated male age-adjusted estimates to the 2000 US standard population ${ }^{11}$ that were not previously ageadjusted due to the small number of cases. ${ }^{7}$ The MLSP rates were initially presented as combined race and ethnicity categories (eg, non-Hispanic white),${ }^{6}$ but for the meta-analysis race and ethnicity rates were recalculated separately for consistency. The number of new SLE cases in the USA was estimated using the pooled age-adjusted sex-specific and race-specific incidence rates from the four states and the age-adjusted AI/ AN population incidence rates from the IHS, which were extrapolated to 2018 US Census population data. 
Table 1 Estimated number of new persons diagnosed with SLE in the USA in 2018

\begin{tabular}{|c|c|c|c|}
\hline & Female & & \\
\hline $\begin{array}{l}\text { Race/ethnicity (number of sites in } \\
\text { the analysis) }\end{array}$ & $\begin{array}{l}\text { SLE incidence* per } \\
100000(95 \% \mathrm{Cl})\end{array}$ & $\begin{array}{l}\text { Population } \\
\text { denominator }\end{array}$ & $\begin{array}{l}\text { Estimated SLE cases in the } \\
\text { USA, } n(95 \% \mathrm{Cl})\end{array}$ \\
\hline Black (4) & 15.9 (12.5 to 20.3$)$ & 24880722 & 3956 (3110 to 5051$)$ \\
\hline White (4) & 5.7 (4.9 to 6.7$)$ & 130137989 & 7418 (6377 to 8719$)$ \\
\hline Total† & 8.7 (8.1 to9.4) & 169802573 & $12560(10325$ to 15402$)$ \\
\hline \multicolumn{4}{|l|}{ Ethnicity } \\
\hline \multirow[t]{2}{*}{ Hispanic $\neq(2)$} & 6.8 (6.2 to 7.6$)$ & 30689083 & 2087 (1903 to 2332) \\
\hline & \multicolumn{3}{|l|}{ Male } \\
\hline Black (4) & 2.4 (1.8 to 3.0$)$ & 22961129 & 551 (413 to 689 ) \\
\hline White (4) & 0.8 (0.6 to 1.1$)$ & 127942583 & 1024 (768 to 1407$)$ \\
\hline Asian/PI (2) & $0.4(0.2$ to 0.6$)$ & 11660533 & 47 (23 to 70$)$ \\
\hline $\mathrm{Al} / \mathrm{AN}(1)$ & 3.8 (1.6 to 7.8$)$ & 2134870 & 81 (34 to 167$)$ \\
\hline Total† & $1.2(0.9$ to 1.6$)$ & 164699115 & 1703 (1238 to 2333 ) \\
\hline \multicolumn{4}{|l|}{ Ethnicity } \\
\hline Hispanicł (2) & $0.9(0.4$ to 1.9$)$ & 31281605 & 282 (125 to 594) \\
\hline
\end{tabular}

SLE cases were defined according to the 1997 revised American College of Rheumatology criteria.

${ }^{*}$ Estimates for black and white persons are based on pooled estimates from the four state-based registries; Asian/PI and Hispanics are based on pooled estimates from California and New York; Al/AN estimates are based on the Indian Health Service registry.

†The pooled 'total' incidence estimate includes black, white and Asian/PI. Since the Al/AN incidence was based on one registry and was significantly higher, it was not included in the pooled incidence per 100000.

¥Hispanic ethnicity is not mutually exclusive from the race categories, that is, all Hispanic persons are included in one of the race categories. Thus, the pooled estimates do not incorporate the Hispanic rates since that would lead to duplicate counting. Estimates for Hispanics are based on pooled estimates from California and New York.

Al/AN, American Indian/Alaska Native; Asian/PI, Asian/Pacific Islander.

The sex-specific and race-specific estimates were then summed for the total population count.

\section{Patient and public involvement}

Neither patients nor the public were involved in the design, or conduct, or reporting or dissemination plans of our research.

\section{RESULTS}

\section{Incidence}

The five registries contributed 1057 SLE cases fulfilling the ACR classification. The random effects model for the metaanalysis of estimates from the four state-based registries yielded an overall SLE incidence rate of 5.1 per 100000 person-years (95\% CI 4.6 to 5.6) (figure 1). The incidence among females was about seven times higher than among males (8.7 vs 1.2) (table 1). From the race-specific and ethnicity-specific pooled estimates from the four statespecific registries, the incidence rate was highest among black females $(15.9,95 \%$ CI 12.5 to 20.3$)$, followed by Asian/Pacific Islander (7.6, 95\% CI 5.5 to 10.4), Hispanic
(6.8, 95\% CI 6.2 to 7.6$)$ and white females $(5.7,95 \%$ CI 4.9 to 6.7 ) (figure $2 \mathrm{~A}$ and table 1 ). Among males, the incidence rate was highest among black males $(2.4,95 \% \mathrm{CI}$ 1.8 to 3.0$)$, followed by Hispanic $(0.9,95 \%$ CI 0.4 to 1.9$)$, white $(0.8,95 \% \mathrm{CI} 0.6$ to 1.1$)$ and Asian/Pacific Islander males $(0.4,95 \%$ CI 0.2 to 0.6$)$ (figure $2 \mathrm{~B}$ and table 1$)$. The age-adjusted incidence rate estimates for the AI/AN population from the IHS registry (which were not included in the pooled meta-analysis estimates from the four statebased registries) were the second highest of all the race/ ethnicity categories for females (10.4, 95\% CI 6.6 to 14.6) and highest for males (3.8, 95\% CI 1.6 to 7.8) (figure 2A,B and table 1).

\section{Number of newly diagnosed SLE cases in the USA}

Applying our sex-specific and race-specific incidence estimates to the corresponding population denominators from 2018 Census data, we estimated that 14263 (95\% CI 11563 to 17735 ; 12560 females and 1703 males) persons in the USA were newly diagnosed with SLE in 2018 (table 1). 

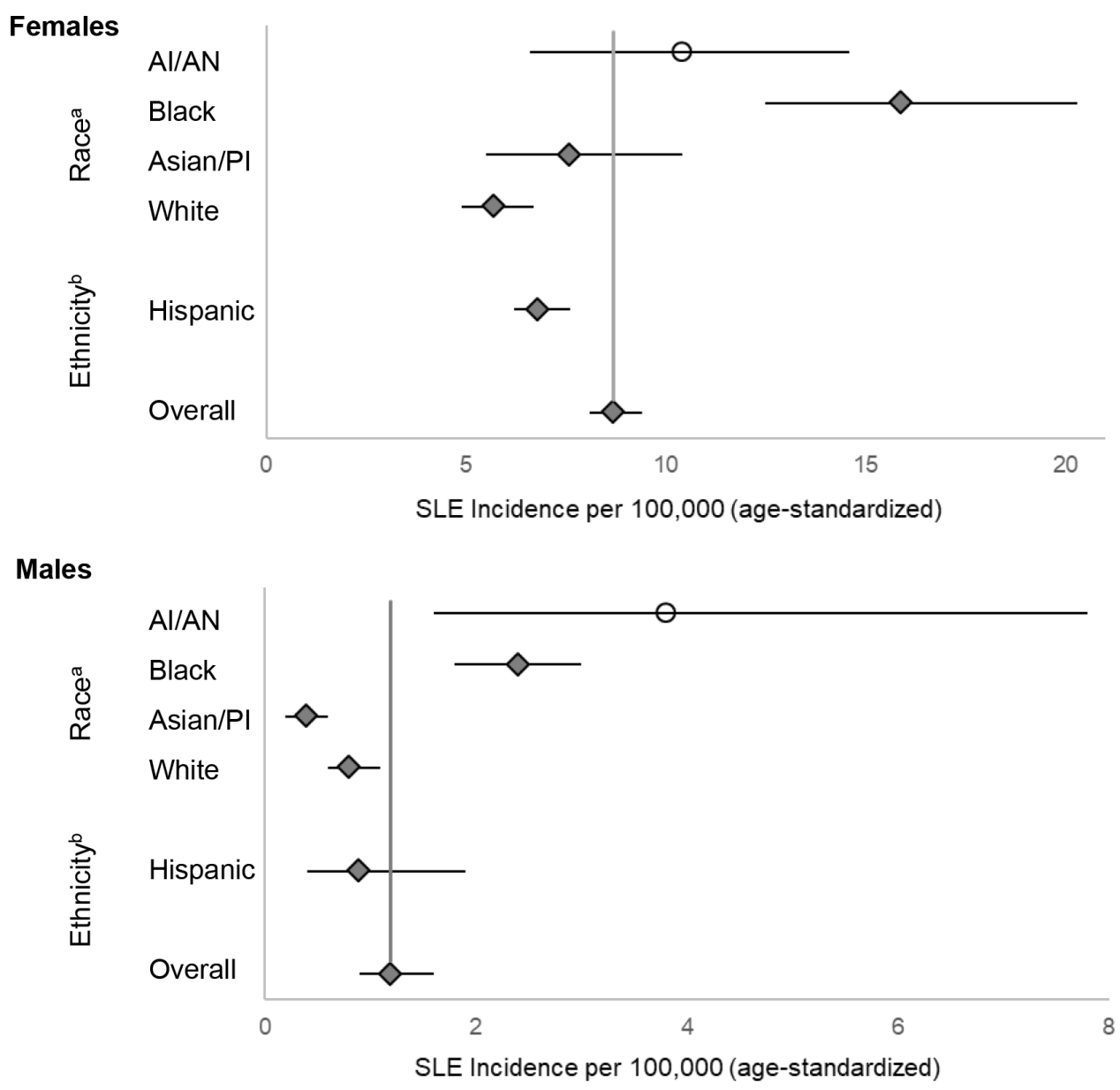

Figure 2 Meta-analysis results of the SLE incidence based on four CDC population-based registries, overall and by race and Hispanic ethnicity among females (A) and males (B). The overall female and male meta-analysis estimate is based on the results from the lupus registry sites in Michigan, Georgia, New York and California. SLE cases were defined according to the 1997

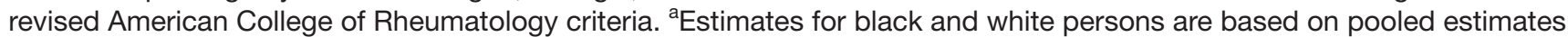
from the four state-based registries; Asian/PI are based on pooled estimates from California and New York. Al/AN age-adjusted incidence estimates are from the Indian Health Service registry data previously published for females ${ }^{7}$ and newly age-adjusted to the 2000 US standard population by us for males..$^{11}$ In the original publication, the rates for males were not age-adjusted

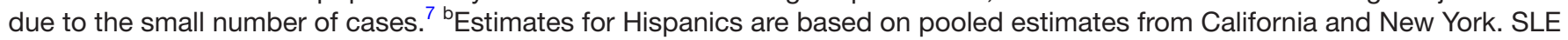
cases were defined according to the American College of Rheumatology criteria. Al/AN, American Indian/Alaska Native; Asian/ PI, Asian/Pacific Islanders; CDC, Centers for Disease Control and Prevention.

\section{DISCUSSION}

The overall SLE incidence in the USA was estimated to be 5.1 per 100000 person-years (95\% CI 4.6 to 5.6) during calendar years 2002-2009. Incidence rates were approximately seven times higher in females compared with males and highest among black and AI/AN females. Extrapolating sex-specific and race-specific estimates to 2018 US Census data, we estimated that 14263 (95\% CI 11563 to 17 735) persons (12560 and 1703 females and males, respectively) in the USA were newly diagnosed with SLE fulfilling ACR classification criteria.

Our analyses have several limitations as previously described $^{3-8}$ There were minor differences in the ICD-9 criteria and case-finding sources used to identify possible cases by the different registries. Capture-recapture analyses conducted by the state-based registries demonstrated small numbers of missing cases meeting the ACR criteria. ${ }^{3-6}$ Undiagnosed cases were not counted, nor were 'incomplete' lupus, drug-induced lupus or primary cutaneous lupus. ${ }^{15} 16$ Race and ethnicity were abstracted from medical records, which may not accurately represent the patient's own racial or ethnic identification. The different races and Hispanic ethnicity encompass heterogeneous subgroups, and SLE rates among these subgroups may differ. The AI/AN incidence rates were based only on the IHS registry ${ }^{7}$ and were not used for the meta-analysis pooled incidence calculations given it focused on one demographic population. However, this registry included three geographical regions with different population characteristics to improve the generalisability of results, and these results were used for the national estimate calculation of new SLE cases. Other SLE case definitions used by the five registries ${ }^{3-7}$ resulted in slightly higher estimates in most instances, although greater sensitivity may have occurred with lower specificity. Finally, our incidence estimates from 2002 to 2004 and from 2007 to 2009 were applied to the 2018 Census population, which 
provides a companion to our 2018 prevalence estimate ${ }^{8}$; both might be slightly affected if lupus incidence or prevalence changed significantly during that period.

Despite these limitations, our analysis has several strengths. The case-finding was population-based and thus likely captured a wider spectrum of SLE than previous studies. The public health surveillance exemption additionally facilitated broad case-finding and data collection. Cases were also validated through standardised and quality-controlled abstracting, with rigid reviews of all available medical records by trained abstractors. Additionally, we used harmonised methods and data dictionaries and defined cases using the 1997 revised ACR SLE classification criteria. ${ }^{9} 10$ The CDC registries included SLE cases from diverse populations across the country with substantial representation of the major racial and ethnic groups found in the USA, allowing for a better understanding of SLE by race/ethnicity as well as among understudied males.

Most importantly, this is the first study to use representative registry data to estimate the incidence of SLE in the USA. We were able to use information from a large, coordinated network of population-based active surveillance registries to estimate an SLE incidence of 5.1 per 100000 person-years in the USA, corresponding to 14263 newly diagnosed persons in 2018. Both likely represent a lower bound for SLE incidence but provide new information to inform future research and policy efforts addressing the burden of this rare disease.

\section{Author affiliations}

${ }^{1}$ Department of Medicine, Division of Rheumatology, New York University Grossman School of Medicine, New York, New York, USA

${ }^{2}$ Department of Research Services, Division of Community Health Services, Alaska Native Tribal Health Consortium, Anchorage, Alaska, USA

${ }^{3}$ University of Michigan Schools of Medicine \& Public Health, Ann Arbor, Michigan, USA

${ }^{4}$ Department of Biostatistics, University of Michigan School of Public Health, Ann Arbor, Michigan, USA

${ }^{5}$ Department of Medicine, Division of Rheumatology, Emory University, Atlanta, GA, USA

${ }^{6}$ University of California, San Francisco, California, USA

${ }^{7}$ Division of Rheumatology, University of Michigan, Ann Arbor, Michigan, USA

${ }^{8}$ Rheumatology Research Group, Institute of Inflammation and Ageing, University of Birmingham, Birmingham, UK

${ }^{9}$ Centers for Disease Control and Prevention, Atlanta, Georgia, USA

${ }^{10}$ New York City Department of Health and Mental Hygiene, New York, New York, USA

Acknowledgements The authors would like to acknowledge Benjamin Wainwright for his assistance in preparing the manuscript.

Contributors $\mathrm{Pl}$ is responsible for the overall content as guarantor. Pl accepts responsibility for the finished work and/or the conduct of the study, had access to the data and controlled the decision to publish.

Funding Support for this analysis was provided by cooperative agreements between the New York City Department of Health and Mental Hygiene and the New York University School of Medicine, and the Centers for Disease Control and Prevention (U01DP006489) and the National Institutes of Health (UL1TR002240) to the University of Michigan. Data collection was funded under the following cooperative agreements: Georgia Lupus Registry: DP08806; Michigan Lupus Epidemiology and Surveillance Program: DP001441; California Lupus Surveillance Program: A114297; Manhattan Lupus Surveillance Program: DP002827; and Indian Health Service: Interagency Agreement Award IAA10FED1003070.
Competing interests None declared.

Patient and public involvement Patients and/or the public were not involved in the design, or conduct, or reporting, or dissemination plans of this research.

Patient consent for publication Not required.

Ethics approval This study does not involve human participants.

Provenance and peer review Not commissioned; externally peer reviewed.

Open access This is an open access article distributed in accordance with the Creative Commons Attribution Non Commercial (CC BY-NC 4.0) license, which permits others to distribute, remix, adapt, build upon this work non-commercially, and license their derivative works on different terms, provided the original work is properly cited, appropriate credit is given, any changes made indicated, and the use is non-commercial. See: http://creativecommons.org/licenses/by-nc/4.0/.

\section{ORCID iDs}

Peter M Izmirly http://orcid.org/0000-0001-5445-2182

Elizabeth D Ferucci http://orcid.org/0000-0003-0478-8205

Emily C Somers http://orcid.org/0000-0001-5234-3978

S Sam Lim http://orcid.org/0000-0003-2361-0787

Cristina Drenkard http://orcid.org/0000-0002-6832-7291

\section{REFERENCES}

1 Lim SS, Drenkard C, McCune WJ, et al. Population-based lupus registries: advancing our epidemiologic understanding. Arthritis Rheum 2009;61:1462-6.

2 Drenkard C, Lim SS. Update on lupus epidemiology: advancing health disparities research through the study of minority populations. Curr Opin Rheumatol 2019;31:689-96.

3 Lim SS, Bayakly AR, Helmick CG, et al. The incidence and prevalence of systemic lupus erythematosus, 2002-2004: the Georgia lupus registry. Arthritis Rheumatol 2014;66:357-68.

4 Somers EC, Marder W, Cagnoli P, et al. Population-based incidence and prevalence of systemic lupus erythematosus: the Michigan lupus epidemiology and surveillance program. Arthritis Rheumatol 2014;66:369-78.

5 Dall'Era M, Cisternas MG, Snipes K, et al. The incidence and prevalence of systemic lupus erythematosus in San Francisco County, California: the California lupus surveillance project. Arthritis Rheumatol 2017;69:1996-2005.

6 Izmirly PM, Wan I, Sahl S, et al. The incidence and prevalence of systemic lupus erythematosus in New York County (Manhattan), New York: the Manhattan lupus surveillance program. Arthritis Rheumatol 2017;69:2006-17.

7 Ferucci ED, Johnston JM, Gaddy JR, et al. Prevalence and incidence of systemic lupus erythematosus in a population-based registry of American Indian and Alaska native people, 2007-2009. Arthritis Rheumatol 2014;66:2494-502.

8 Izmirly PM, Parton H, Wang L, et al. Prevalence of systemic lupus erythematosus in the United States: estimates from a meta-analysis of the centers for disease control and prevention national lupus registries. Arthritis Rheumatol 2021;73:991-6.

9 Hochberg MC. Updating the American College of rheumatology revised criteria for the classification of systemic lupus erythematosus. Arthritis \& Rheumatism 1997;40:1725.

10 Tan EM, Cohen AS, Fries JF, et al. The 1982 revised criteria for the classification of systemic lupus erythematosus. Arthritis Rheumatol 1982;25:1271-7.

11 National Cancer Institute, National Institutes of Health. Standard populations (millions) for Age-Adjustment, 2019. Available: https:// seer.cancer.gov/stdpopulations/

12 Huedo-Medina TB, Sánchez-Meca J, Marín-Martínez F, et al. Assessing heterogeneity in meta-analysis: Q statistic or 12 index? Psychol Methods 2006;11:193-206.

13 Higgins JPT, Thompson SG. Quantifying heterogeneity in a metaanalysis. Stat Med 2002;21:1539-58.

14 Balduzzi S, Rücker G, Schwarzer G. How to perform a meta-analysis with R: a practical tutorial. Evid Based Ment Health 2019;22:153-60.

15 Drenkard C, Parker S, Aspey LD, et al. Racial disparities in the incidence of primary chronic cutaneous lupus erythematosus in the southeastern us: the Georgia lupus registry. Arthritis Care Res 2019;71:95-103.

16 Izmirly P, Buyon J, Belmont HM, et al. Population-based prevalence and incidence estimates of primary discoid lupus erythematosus from the Manhattan lupus surveillance program. Lupus Sci Med 2019;6:e000344. 